\title{
Polarization-Singular Structure of Laser Images of Stratified Phase-Inhomogeneous Layers for the Diagnostics and Classification of their Optical Properties
}

\author{
Yu. A. Ushenko ${ }^{a}$, I. Z. Misevich ${ }^{b}$, O. Yu. Telenga ${ }^{b}$, Yu. Ya. Tomka ${ }^{b}$, and A. O. Karachevtsev \\ ${ }^{a}$ Correlation Optics Department, Chernivtsi National University, 2 Kotsyubinsky Str., 58012, Chernivtsi, Ukraine \\ ${ }^{b}$ Optics and Spectroscopy Department, Chernivtsi National University, 2 Kotsyubinsky Str., 58012, Chernivtsi, Ukraine \\ e-mail: yuriyu@gmail.com
}

Received November 22, 2010; in final form, December 8, 2010

\begin{abstract}
Presented in this work are the results of investigation aimed at analysis of coordinate distributions for azimuths and ellipticity of polarization (polarization maps) in laser images of three types of phase-inhomogeneous layers, namely: rough, ground and bulk scattering layers. To characterize polarization maps for all the types of phase-inhomogeneous layers, the authors have offered to use three groups of parameters: statistical moments of the first to the fourth orders, autocorrelation functions and logarithmic dependences for power spectra related to distributions of azimuths and ellipticity of polarization inherent to phase-inhomogeneous layers laser images. Ascertained are the criteria for diagnostics and classification of phase-inhomogeneous layers optical properties.
\end{abstract}

Keywords: polarization, rough surface, phase-inhomogeneous layer, statistic moments, autocorrelation, polarization singularity.

DOI: $10.3103 / \mathrm{S} 1060992 X 11010048$

\section{INTRODUCTION}

By tradition, the processes of transforming optical radiation of phase-inhomogeneous objects and media are considered, as a rule, in a statistical approach (theory of radiation transfer [1], Monte Carlo modeling [2]). Among the most spread traditional methods for studying the scattered light fields, one can separate the following independent directions: "scalar" (photometry and spectrophotometry) $[3,4]$ and "vector" (polarization nephelometry, Mueller-matrix optics) [5-13]. Using these approaches, determined are interrelations between the sets of statistical moments of the 1st to the 4th orders, correlation functions, fractal dimensionalities that characterize phase-inhomogeneous or rough surfaces and coordinate distributions for phases, azimuths and ellipticity of polarization in their laser images [14-22].

In parallel with traditional statistical investigations, formed in recent 10 to 15 years is the new optical approach to describe a structure of polarizationally inhomogeneous fields in the case of scattered coherent radiation. The main feature of this approach is the analysis of definite polarization states to determine the whole structure of coordinate distributions for azimuths and ellipticities of polarization. The so-called polarization singularities are commonly used as these states [23-37]:

- states with linear polarization when the direction of rotation for the electric field vector is indefinite, the so-called $L$-points;

- circularly-polarized states when the azimuth of polarization for the electric field vector is indefinite, the so-called $C$-points.

This work is aimed at ascertaining the possibilities to diagnose and classify the human skin phase-inhomogeneous layers (PhILS) of various types (surface-scattering — superficial epithelium layer, subsurfacescattering - stratified epithelium structures and bulk-scattering ones-dermal layer) by determination values and ranges for changing the statistical (moments of the 1st to the 4th orders), correlation (autocorrelation functions) and fractal (logarithmic dependences for power spectra) parameters that characterize coordinate distributions for polarization-singular states in PhILS laser images. 


\section{MAIN MODEL CONCEPTIONS AND ANALYTICAL RELATIONS}

As a base for analytical description of processes providing formation of polarization-inhomogeneous images for various types of PhILS, we have used the model conceptions developed in the works [5, 14, 19, 21, 22]:

- surface-scattering PhILS is a rough surface consisting of an ensemble of quasi-plane, chaotically oriented micro-areas of optical anisotropic epithelium with optical dimensions $l>\lambda$ - "group 1 " or PhILS-1;

- PhILS with surface and subsurface scattering — the superposition of superficial epithelium layer and subsurface net of collagen fibrils - "group 2" or PhILS-2;

—PhILS with bulk scattering — bulk collagen net of skin dermal layer of a various optical thickness"group 3" or PhILS-3.

\subsection{Mechanisms Providing Formation of Polarization-Inhomogeneous Images for PhILS-1}

As an example of PhILS with surface scattering, we have considered the optically thin (the attenuation coefficient $\tau<0.1)$ histological sections of a superficial skin epithelium layer. The range of changes in geometric sizes $(l)$ of micro-roughness (micro-areas) for these rough surfaces corresponds to 2 to $60 \mu \mathrm{m}$. Optical properties of each micro-area are exhaustively characterized with the Jones operator of the following form

$$
\{R\}=\left\|\begin{array}{cc}
1 & 0 \\
0 & p_{y} / p_{x}
\end{array}\right\| .
$$

It is possible to show that within the sizes $(\Delta x, \Delta y)$ of one micro-area there takes place the change of polarization azimuth $\alpha$ inherent to the refracted plane-polarized laser wave with the initial azimuth $\alpha_{0}$

$$
\alpha(\Delta x, \Delta y)=\arctan \frac{p_{y} U_{0 y}}{p_{x} U_{0 x}}=\arctan \left[\frac{p_{y}}{p_{x}} \tan \alpha_{0}\right],
$$

where $U_{0 x}, U_{0 y}$ are orthogonal components of the amplitude $U_{0} ; p_{x}, p_{y}$-Fresnel amplitude coefficients for transmission [5].

Thus, in the approach of single scattering the polarization image of rough surface may be considered as coordinate-distributed parts of $L$-polarized states.

\subsection{Mechanisms Providing Formation of Polarization-Inhomogeneous Images for PhILS-2}

As examples of these PhILS, we have considered more optically thick (the attenuation coefficient $\tau=$ 0.2-0.4) skin histological sections - superficial epithelium layer and subsurface dermal layer.

The process providing formation of a local polarization state can be considered as superposition of "influences" of superficial epithelium layer and subsurface net of optical-anisotropic collagen fibrils. From analytical viewpoint, this scenario can be described by superposition $\{F\}$ of the Jones matrix operators for these partial layers (cracked $\{T\}$ and surface $\{R\}$ )

$$
\begin{gathered}
\{F\}=\{R\}\{T\}=\left\|\begin{array}{l}
f_{11} f_{12} \\
f_{21} f_{22}
\end{array}\right\|=\left\|\begin{array}{c}
\left(r_{11} t_{11}+r_{12} t_{21}\right) ;\left(r_{11} t_{12}+r_{12} t_{22}\right) ; \\
\left(r_{21} t_{11}+r_{22} t_{21}\right) ;\left(r_{21} t_{12}+r_{22} t_{22}\right)
\end{array}\right\|, \\
\{T\}=\left\|\begin{array}{ll}
t_{11} & t_{12} \\
t_{21} & t_{22}
\end{array}\right\|=\left\|\begin{array}{c}
\left.\cos ^{2} \gamma+\sin ^{2} \gamma \exp (i \delta)\right] ; \quad[\cos \gamma \sin \gamma \exp (i \delta)] ; \\
{[\cos \gamma \sin \gamma \exp (i \delta)] ; \quad\left[\sin ^{2} \gamma+\cos ^{2} \gamma \exp (i \delta)\right]}
\end{array}\right\| .
\end{gathered}
$$

Here, $\gamma$ is the direction of the optical axis inherent to crystalline fibril; $\delta$ - phase shift between orthogonal components $\left(U_{x} ; U_{y}\right)$ of the amplitude $(U)$ of laser wave with the wavelength $\lambda$ caused by birefringence in the matter $\Delta n$. 
If taking into account the relations (1), (3) and (4), it follows that within the limits $(\Delta x, \Delta y)$ of a local bulk created by the micro-area and crystalline fibril, formed is an elliptically polarized part of the object field with the following parameters

$$
\begin{aligned}
& \tilde{\alpha}(\Delta x, \Delta y)=\arccos \left(\frac{\sin \delta}{\cos 2\left(\arctan \left\{\left[\frac{\left(f_{21}+f_{22}\right)^{2}}{\left(f_{11}+f_{12}\right)^{2}}\right] \tan \alpha_{0}\right\}\right)}\right) \\
& \tilde{\beta}(\Delta x, \Delta y)=\arcsin \left(\frac{\tan \delta}{\sin 2\left(\arctan \left\{\left[\frac{\left(f_{21}+f_{22}\right)^{2}}{\left(f_{11}+f_{12}\right)^{2}}\right] \tan \alpha_{0}\right\}\right)}\right)
\end{aligned}
$$

As it follows from the analytical relations (5) and (6), interaction of the plane-polarized $\left(\alpha_{0}\right)$ wave with the PhIL of this type provides formation of a polarization-inhomogeneous laser image. Among the whole set of values $(\tilde{\alpha} ; \tilde{\beta})$, formation of $L$ and $\pm C$ polarization states seems to be very probable

$$
\begin{gathered}
L-\Leftrightarrow \delta(\Delta x, \Delta y)=q \pi, \quad q=1,2, \ldots \\
\pm C-\Leftrightarrow \tan \delta(\Delta x, \Delta y)=\sin 2\left(\arctan \left\{\left[\frac{\left(f_{21}(\Delta x, \Delta y)+f_{22}(\Delta x, \Delta y)\right)^{2}}{\left(f_{11}(\Delta x, \Delta y)+f_{12}(\Delta x, \Delta y)\right)^{2}}\right] \tan \alpha_{0}\right\}\right) .
\end{gathered}
$$

\subsection{Mechanisms Providing Formation of Polarization-Inhomogeneous Images for PhILS-3}

When analyzing the processes of interaction of laser radiation with these PhILS-3 (bulk collagen net of skin dermal layer, the attenuation coefficient $\tau>0.4$ ), we have used the method of superposition of the Jones matrix operators ( 3 ) for the set of sequentially disposed optically-thin layers

$$
\{\Phi\}=\left\{\Phi^{(p)}\right\}\left\{\Phi^{(p-1)}\right\} \times \ldots \times\left\{\Phi^{(1)}\right\} .
$$

Having calculated the set of Jones matrix elements $\phi_{g g}$ for an optically-thick PhILS-3, one can define analytical expressions (like to (5) $-(6))$ to find $L$ and $\pm C$ polarization states in the laser image

$$
\begin{gathered}
L-\Leftrightarrow \delta^{*}(\Delta x, \Delta y)=q \pi, \quad q=1 ; 2, \ldots ; \\
\pm C-\Leftrightarrow \tan \delta^{*}(\Delta x, \Delta y)=\sin 2\left(\arctan \left\{\left[\frac{\left(\phi_{21}(\Delta x, \Delta y)+\phi_{22}(\Delta x, \Delta y)\right)^{2}}{\left(\phi_{11}(\Delta x, \Delta y)+\phi_{12}(\Delta x, \Delta y)\right)^{2}}\right] \tan \alpha_{0}\right\}\right) .
\end{gathered}
$$

Thus, the above analytical consideration (relations (1) to (11)) for various scenarios of transformation of laser radiation by PhILS in all the cases enabled to reveal the principled possibility of formation of polarization-singular states $(\beta=0 ; \beta= \pm \pi / 4)$ in respective laser images.

\section{EXPERIMENTAL SETUP FOR POLARIMETRIC INVESTIGATIONS}

Our study of polarization-inhomogeneous laser images inherent to PhILS was performed using the optical scheme of a laser polarimeter (Fig. 1) [38].

Illumination was performed using a parallel beam $\left(\varnothing=10^{4} \mu \mathrm{m}\right)$ from an $\mathrm{He}-\mathrm{Ne}$ laser $(\lambda=0.6328 \mu \mathrm{m})$ 1. The polarization illuminator (quarter-wave plates 3 and 5 as well as polarizer 4 ) were used to form various polarization states in the laser beam. Polarization images of PhILS 6 were projected using the microobjective 7 into the plane of the light-sensitive area $(800$ pix $\times 600$ pix $)$ in CCD camera 10 . Turning the transmission axis of the analyzer 9 by the angles $\pm 45^{\circ}$ relatively to the direction of the highest velocity axis for the quarter-wave plate 8 , wee could determine the intensities of right $\left(I_{\otimes}\right)$ and left $\left(I_{\oplus}\right)$ circularly polarized components for each separated pixel of CCD camera 10. It served as a base to calculate coordinate 


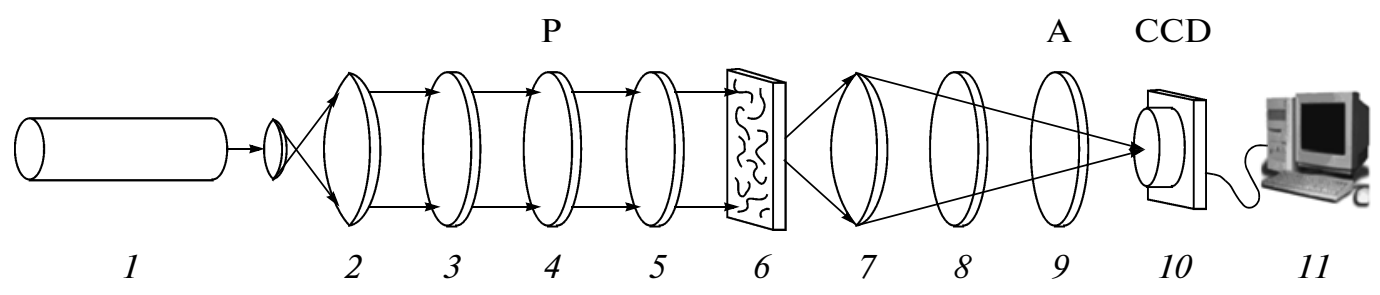

Fig. 1. Optical scheme of the polarimeter: $1-\mathrm{He}-\mathrm{Ne}$ laser; 2 - collimator; 3, 5, 8-quarter-wave plates; 4, 9-polarizer and analyzer, respectively; 6 - object under investigation; 7-micro-objective; 10 -CCD camera; 11 - personal computer.

distributions of the fourth parameter in the Stokes vector $\left.V_{4}(m \times n)\right)$ describing the laser image of PhILS, if using the relation $V_{4}\left(r_{n m}\right)=I_{\otimes}\left(r_{i k}\right)-I_{\oplus}\left(r_{i k}\right) / I_{\otimes}\left(r_{i k}\right)+I_{\oplus}\left(r_{i k}\right)$.

In this work, to describe coordinate $(x, y)$ distributions for polarization-singular $(L, \pm C)$ states in laser images for all the types of PhILS

$$
\left\{\begin{array}{c}
V_{4}(x, y)=0 \leftrightarrow L(\beta=0) ; \\
V_{4}(x, y)= \pm 1 \leftrightarrow \pm C(\beta= \pm \pi / 4) .
\end{array}\right.
$$

The two-dimensional array (12) was scanned along the horizontal direction $x \equiv 1, \ldots, m$ with the step $\Delta x=1$ pix. Within the limits of each local sample $\left(1_{\text {pix }} \times n_{\text {pix }}\right)^{(k=1,2, \ldots, m)}$, we calculated the amount $(N)$ of characteristic values $V_{4}(k)=0,-\left(N_{L}^{(k)}\right)$ and $V_{4}(k)= \pm 1,-\left(N_{ \pm C}^{(k)}\right)$.

Thus, we determined the dependences $N_{L}(x) \equiv\left(N_{L}^{(1)}, N_{L}^{(2)}, . ., N_{L}^{(m)}\right)$ and $N_{ \pm C}(x) \equiv\left(N_{ \pm C}^{(1)}, N_{ \pm C}^{(2)}, \ldots\right.$, $N_{ \pm C}^{(m)}$ ) for amounts of polarization-singular $L$ and $\pm C$-points within the limits of a laser image for PhILS.

\section{CRITERIA TO ESTIMATE POLARIZATION-INHOMOGENEOUS IMAGES OF PHILS}

Distributions $N_{L ; \pm C}(x)$ for the amount of polarization-singular states in laser images of PhILS are characterized with the set of statistical moments of the 1st to the 4th orders $Z_{j=1 ; 2 ; 3 ; 4}$ calculated using the following relations [14]

$$
\begin{aligned}
Z_{1}=\frac{1}{M} \sum_{i=1}^{M}\left|N_{L ; \pm C}^{(i)}(x)\right| ; \quad Z_{2} & =\sqrt{\frac{1}{M} \sum_{i=1}^{M} N_{L ; \pm C}^{(i)}(x)^{2}} ; \quad Z_{3}=\frac{1}{Z_{2}^{3}} \frac{1}{M} \sum_{i=1}^{M} N_{L ; \pm C}^{(i))}(x)^{3} ; \\
Z_{4} & =\frac{1}{Z_{2}^{4}} \frac{1}{M} \sum_{i=1}^{M} N_{L ; \pm C}^{(i)}(x)^{4},
\end{aligned}
$$

where $M=800 \times 600$ is the amount of pixels in CCD camera 10 (Fig. 1 ).

Our analysis of the coordinate structure for $N_{L ; \pm C}(x)$ distributions was based on the autocorrelation method by using the function $[15,19]$

$$
K_{L ; \pm C}(m)=\frac{1}{(n-m) \sigma^{2}} \sum_{t=1}^{n-m}\left[X_{t}-\mu\right]\left[X_{t+m}-\mu\right] .
$$

Here, $n$ is the length of discrete sampling $N_{L ; \pm C}(x)=X_{1}, X_{2}, \ldots, X_{n} ; \mu$-average value, $\sigma^{2}$ - the dispersion; $m, n$ are positive integers.

As correlation parameters that characterize the dependences $K_{L ; \pm C}(x)$, we chose:

- correlation area $S_{L ; \pm C}$

$$
S_{L ; \pm C}=\int K_{L ; \pm C}(m) d m
$$



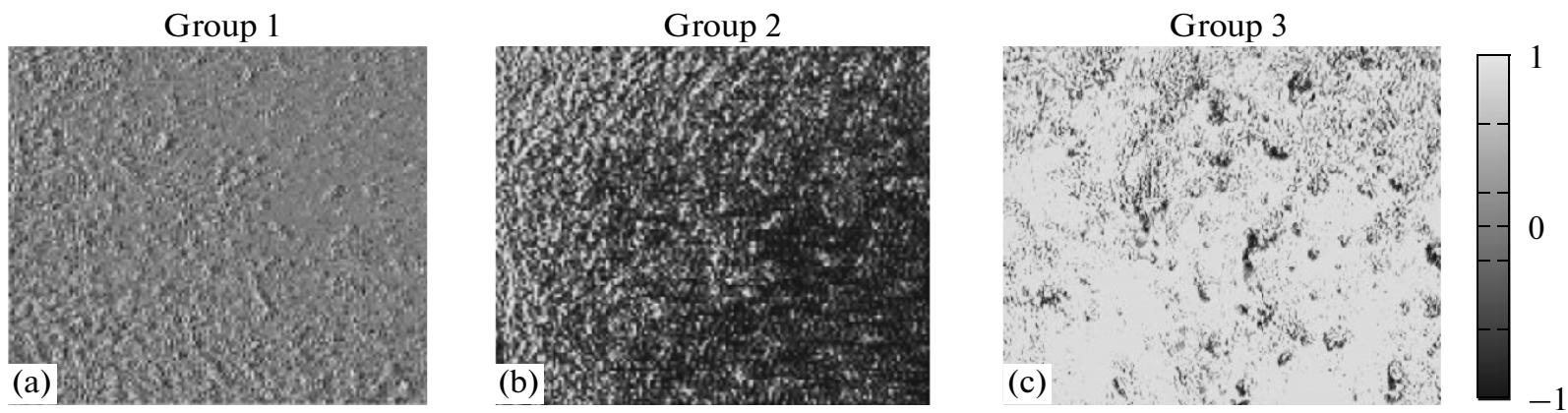

Fig. 2. Coordinate distributions $V_{4}(m \times n)$ of laser images inherent to PhILS: (a) superficial skin epithelium layer; (b) superficial epithelium layer and subsurface dermal layer; (c) bulk collagen net of skin dermal layer $(\tau=1.5)$.

Correlation moment $Q_{L ; \pm C}$ that define the excess for the distribution of values $K_{L ; \pm C}(m)$

$$
Q=\frac{N}{\left(\sum_{i=1}^{N}\left(K_{L ; \pm C}(m)\right)_{i}^{2}\right)^{2}} \sum_{i=1}^{N}\left(K_{L ; \pm C}(m)\right)_{i}^{4}
$$

The fractal analysis of the distributions $N_{L ; \pm C}(x)$ was performed using the calculation of logarithmic dependences $\log J\left[N_{L ; \pm C}(x)\right]-\log d^{-1}$ for the power spectra $J\left\lfloor N_{L ; \pm C}(x)\right\rfloor$, which was calculated as a discrete Fourier transform of the corresponding autocorrelation function $K_{L ; \pm C}(m)$ using the MatLab software

$$
J\left[N_{L ; \pm C}(x)\right]=S_{x x}(w)=\sum_{m=1}^{n} K_{L ; \pm C}(m) e^{-j \omega m},
$$

where $w=d^{-1}$ are the spatial frequencies that are determined by geometrical sizes $(d)$ of PhILS structural elements.

The dependences $\log J\left[N_{L ; \pm C}(x)\right]-\log d^{-1}$ are approximated using the least-squares method into the curves $\Phi(\eta)$, straight parts of which serve to determine the slope angles $\eta$ and calculate fractal $F$ dimensionalities by using the relations [21]

$$
F_{L ; \pm C}=3-\tan \eta \text {. }
$$

Classification of coordinate distributions $N_{L ; \pm C}(x)$ should be performed using the following criteria [22]:

- they are fractal on the condition of a constant slope angle value $\eta=$ const for 2 to 3 decades of changing sizes $d$;

- they are multi-fractal, if several slope angles $\Phi(\eta)$ are available;

- they are random when any stable slope angles are absent within the whole range of changing sizes $d$.

In the latter case, the distributions $\log J\left[N_{L ; \pm C}(x)\right]-\log d^{-1}$ are characterized with the dispersion

$$
D_{z}=\sqrt{\frac{1}{m} \sum_{i=1}^{m}\left[\log J\left(N_{L ; \pm C}\left(x_{i}\right)\right)-\log d^{-1}\right]^{2}} .
$$

\section{STATISTICAL, CORRELATION AND FRACTAL ANALYSIS FOR DISTRIBUTIONS OF POLARIZATION-SINGULAR STATES IN LASER IMAGES OF PhILS 1 TO 3}

Figure 2 illustrates coordinate $(100$ pix $\times 50$ pix) distributions of the fourth parameter for the Stokes vector $V_{4}(m \times n)$ inherent to laser images of PhILS in all the groups $1-3$.

Our qualitative analysis of coordinate distributions $V_{4}(m \times n)$ for laser images of PhILS (Fig. 2) enabled to reveal:

-Practically all the images of the PhILS-1 (Fig. 2a) are linearly polarized field $V_{4}(m \times n)=0$ (relations (2) and (3)). Availability of a small amount of the parts $V_{4}(m \times n) \neq 0$ polarized otherwise can be 
related with interferential effects of multiple interaction of coherent waves with adjacent micro-roughnesses.

- The image of the PhILS-2 (Fig. 2b) is characterized with a developed polarization-inhomogeneous structure formed both by linearly $\left(V_{4}(m \times n)=0\right)$ and elliptically $\left(V_{4}(m \times n) \neq 0\right)$ polarized states, including the circularly $\left(V_{4}(m \times n)=1\right)$ polarized ones (relations (5) to (8)).

- The images of the PhILS-3 are characterized with the widest range of changing the ellipticity of polarization due to multiple bulk scattering (relations (10) and (11)), $--1 \leq V_{4}(m \times n) \leq 1$.

\subsection{States of Laser Images}

Summarized in Fig. 3 is a series of coordinate $\left(V_{4}(m \times n)=0\right)$, quantitative $\left(N_{L}(x)\right)$, autocorrelation $\left(K_{L}(\Delta m)\right)$ and $\operatorname{logarithmic}\left(\log J_{L}-\log d^{-1}\right)$ distributions for polarization-singular $L$ states in laser images of PhILS 1-3.

Our comparative analysis of the obtained dependences $N_{L}(x)$ for the amount of polarization-singular $L$ states in laser images of PhILS in all the groups $1-3$ (Figs. $3 \mathrm{~d}-3 \mathrm{~m}$ ) revealed similarity of their statistical, correlation and fractal structures. For instance, all the $N_{L}(x)$ distributions are close to the equiprobable ones-the condition $Z_{j=3 ; 4}^{L} \ll Z_{j=1 ; 2}^{L}$ is valid for the values of statistical moments (relation (14)). Distinctions between the distributions of $L$ states in laser images of various PhILS are observed as variations of the 1-st and the 2-nd statistical moments $-Z_{1}^{L}=0.61 ; Z_{2}^{L}=0.13 ; Z_{3}^{L}=0.04 ; Z_{4}^{L}=0.09$ (group 1); $Z_{1}^{L}=0.39 ; Z_{2}^{L}=0.21 ; Z_{3}^{L}=0.06 ; Z_{4}^{L}=0.076$ (group 2 ) and $Z_{1}^{L}=0.19 ; Z_{2}^{L}=0.32 ; Z_{3}^{L}=0.03 ; Z_{4}^{L}=0.084$ (group 3).

As seen, for PhILS of the 1-st, 2-nd and 3-rd groups, the mean value $Z_{1}^{L}$ is 1.45 and 3.2 times decreased. And vice versa, the dispersion $Z_{2}^{L}$ is 1.51 and 2.45 times increased. These changes in $N_{L}(x)$ distributions are related with the mechanisms of optically-anisotropic (group 2) and interferential phase modulation (group 3) as well as formation (relations (6) and (10)) of elliptically polarized states in laser images of PhILS. Due to these processes, the total amount of polarization $L$ states is decreased with simultaneous conservation of their equiprobable disposition in the image plane of various PhILS.

The investigated statistical structure of $L$ states for polarization of PhILS laser images is confirmed by a monotonous drop of dependences for autocorrelation functions $K_{L}(\Delta m)$ (relations (15)) of all the distributions $N_{L}(x)$ (Figs. $3 \mathrm{~g}, 3 \mathrm{~h}, 3 \mathrm{~m}$ ). In this case, values of the correlation area $S$ and correlation moment $Q$ trend to their boundary values $\left(S^{L} \longrightarrow 0.33 ; Q^{L} \longrightarrow 0\right.$, relations (16) and (17)) that are characteristic just to equiprobable distributions: $S^{L}=0.26 ; Q^{L}=0.085$ (group 1$) ; S^{L}=0.23 ; Q^{L}=0.14($ group 2$) ; S^{L}=0.2$; $Q^{L}=0.175$ (group 3).

The performed analysis of $\operatorname{logarithmic}$ dependences $\log J_{L}-\log d^{-1}$ (Figs. 3n, 31, 3o) for the power spectra $J\left(N_{ \pm C}\right)$ (relation (18) of the distribution $N_{ \pm C}(x)$ inherent to laser images of the PhILS-1, of the PhILS-2 and PhILS-3 revealed a common regularity - the approximating curves are characterized with stable slope angles that are corresponded with increasing by their value fractal dimensionalities (relation (19)): $F^{L}=2.03$ (group 1); $F^{L}=2.13$ (group 2) and $F^{L}=2.31$ (group 3).

\section{2. $\pm C$ States of Laser Images}

Summarized in Fig. 4 is the series of coordinate $\left(V_{4}(m \times n)=1\right)$, quantitative $\left(N_{ \pm C}(x)\right)$, autocorrelation $\left(K_{ \pm C}(m)\right)$ and $\operatorname{logarithmic}\left(\log J_{ \pm C}-\log d^{-1}\right)$ distributions for polarization-singular $\pm C$-states in laser images of PhILS 2 and 3.

In the laser image of the PhILS-1 $\pm C$-states of polarization are absent (Fig. 4 , left column), which corresponds to model conceptions of mechanisms providing transformation of laser radiation by the set of chaotically oriented micro-areas of the rough surface (relations (1) and (2)).

Optical manifestations of the anisotropic fibrillar layer are illustrated with the network of $\pm C$-points in the laser image of PhILS-4 (Fig. 4a). It is seen that the total amount of circularly polarized points (Fig. 4b) is practically one order less than the amount of linearly polarized states (Figs. 4c, 4d). All the static moments $Z_{j=1 ; 2 ; 3 ; 4}^{ \pm C}$ that characterize the distribution $N_{ \pm C}(x)$ of the amount of circularly polarized singu- 
Group 1

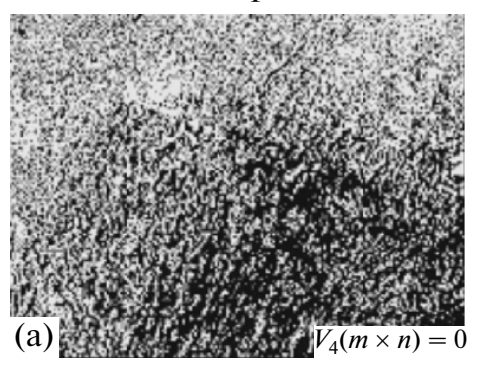

(d)
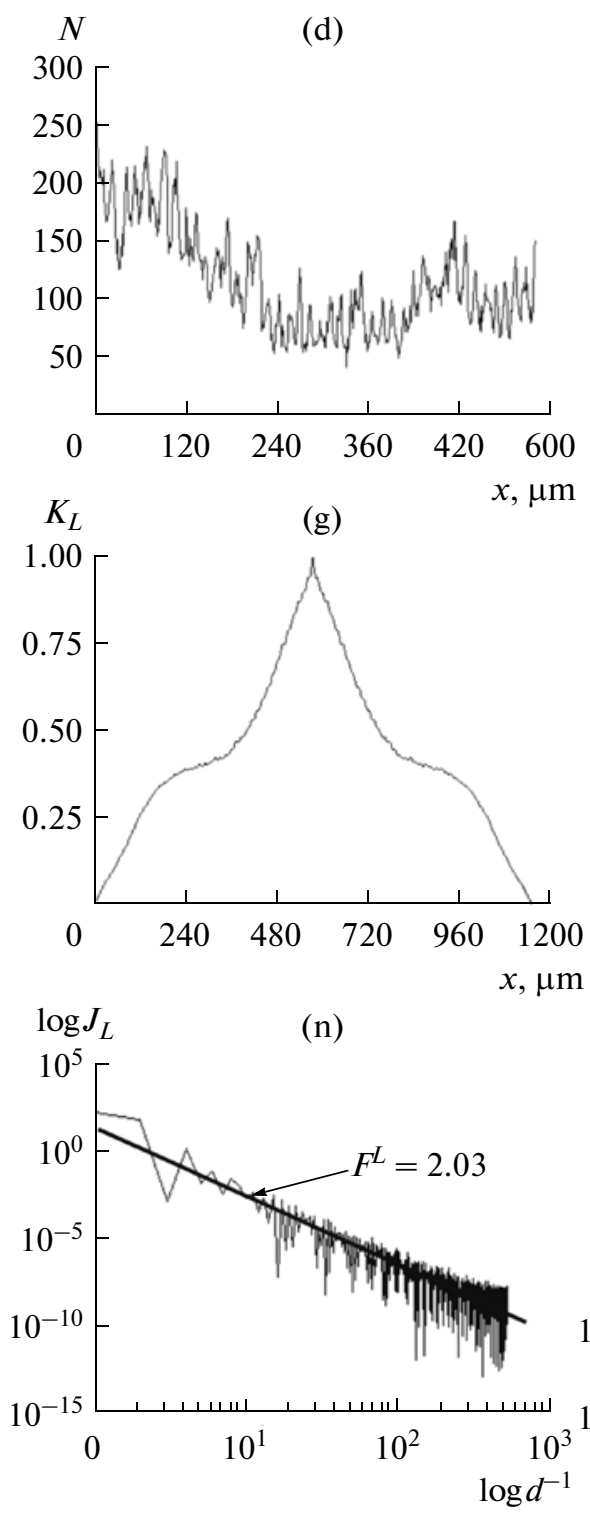

Group 2

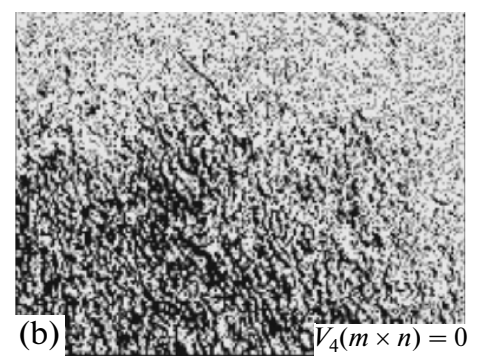

(e)

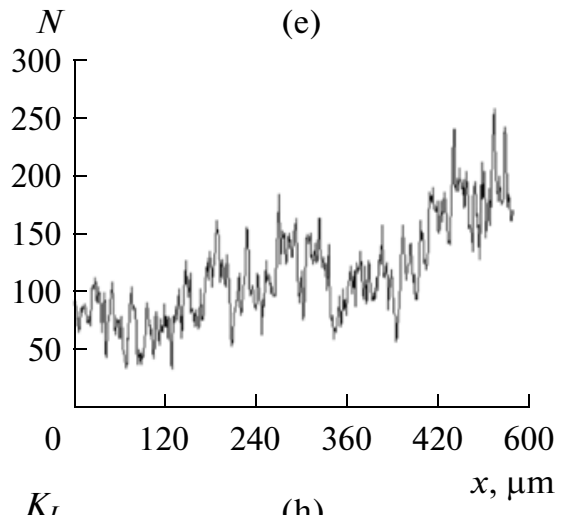

Group 3
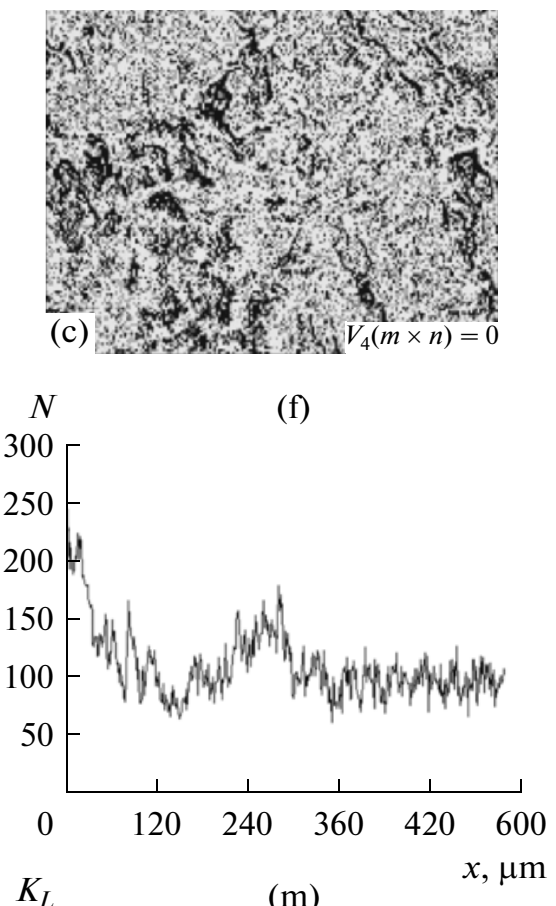
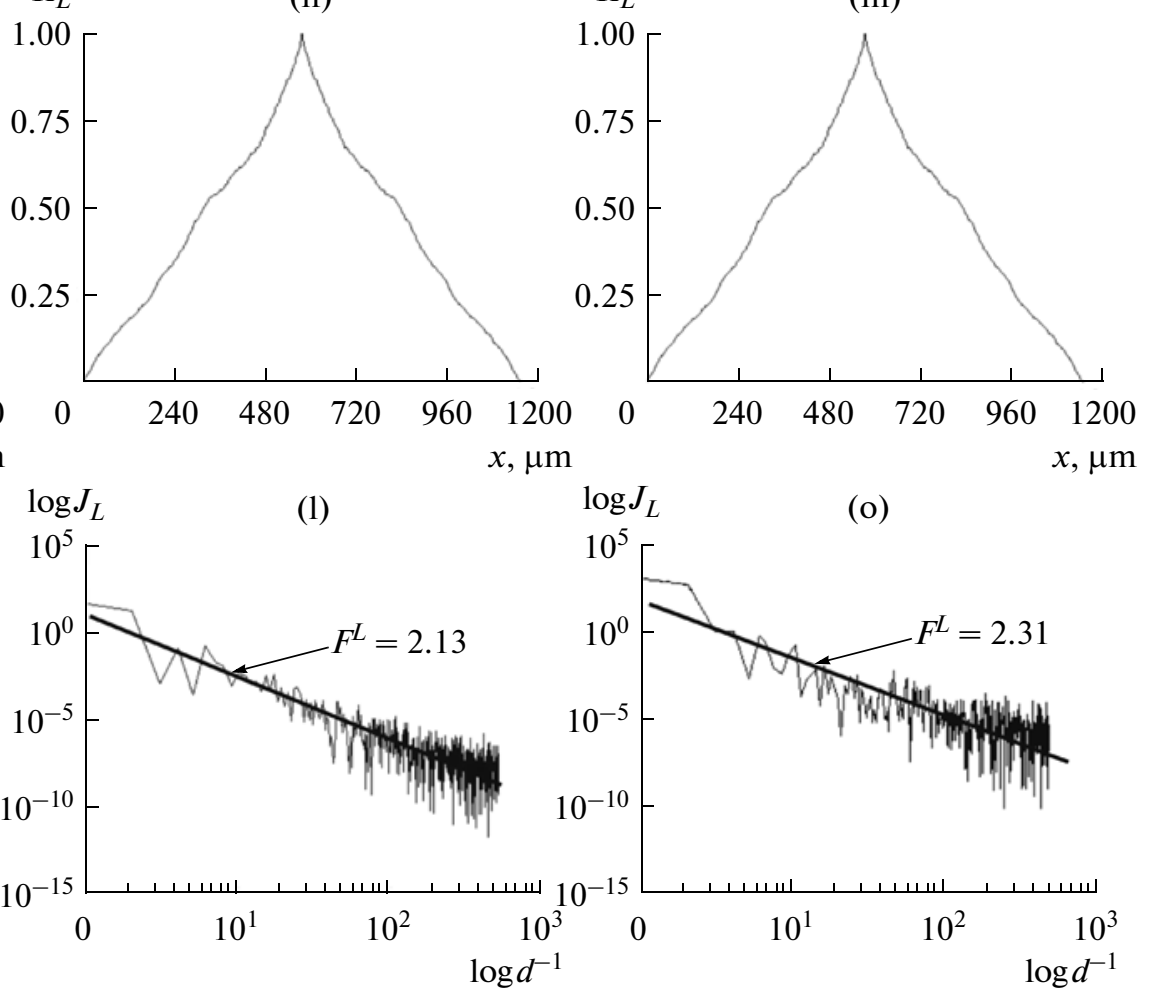

$\log J_{L}$

(o)

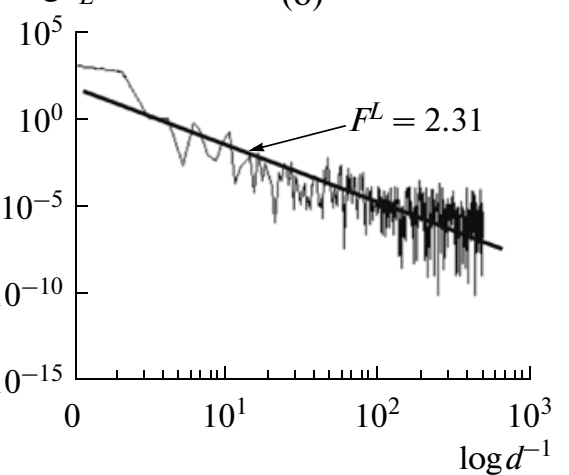

Fig. 3. Coordinate $V_{4}(m \times n)=0\left((\mathrm{a})\right.$, (b), (c)) and quantitative $N_{L}(x)$ distributions ((d), (e), (f)) of $L$ states of polarization; autocorrelation functions $K_{L}(\Delta m)((\mathrm{g}),(\mathrm{h}),(\mathrm{m}))$ and $\log J_{L}-\log d^{-1}$ dependencies $((\mathrm{n}),(\mathrm{l}),(\mathrm{o}))$ for power spectra $J\left(N_{L}\right)$ of the distribution $N_{L}(x)$ for laser images of the different types of PhILS 1 to 3.

lar states differ from zero: $Z_{1}^{ \pm C}=0.093 ; Z_{2}^{ \pm C}=0.35 ; Z_{3}^{ \pm C}=0.42 ; Z_{4}^{ \pm C}=0.45$. In this case, the values of statistical moments of higher orders $Z_{j=2 ; 3 ; 4}^{ \pm C}$ are commensurable: $Z_{2}^{ \pm C} \approx Z_{3}^{ \pm C} \approx Z_{4}^{ \pm C}$. 
Group 1

(absent)

(a)
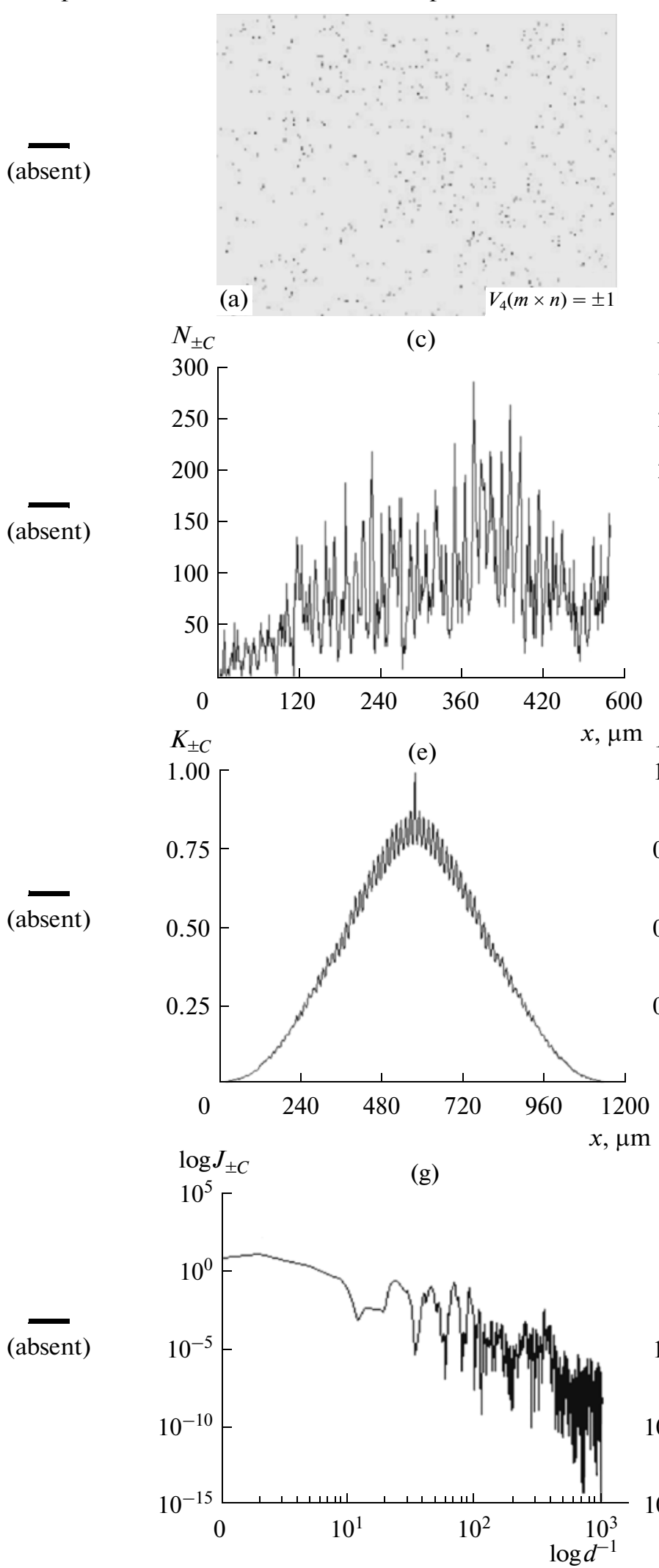

Group 3

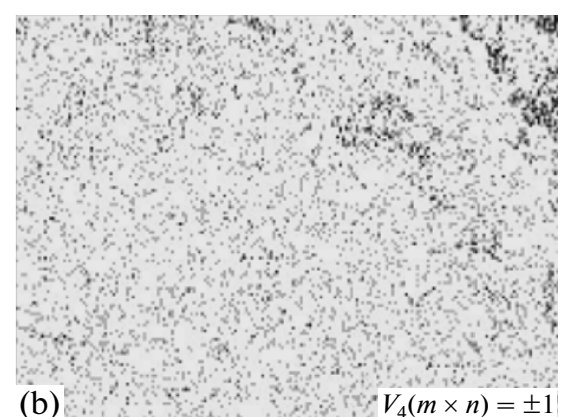

(d)

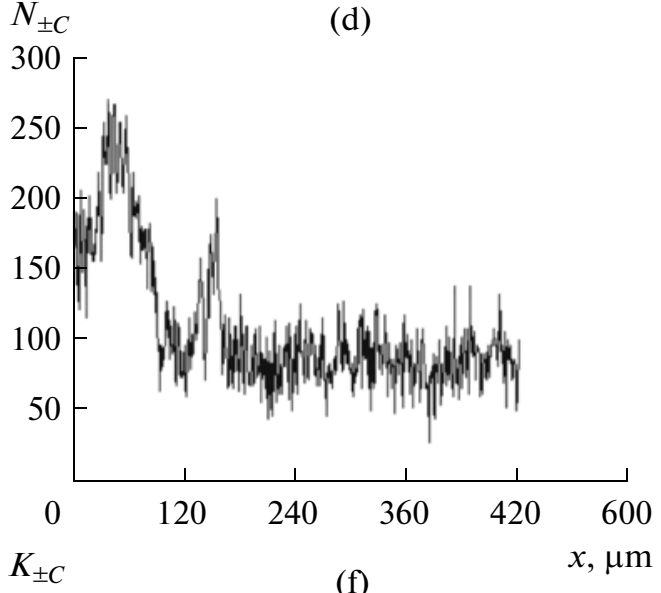

$K_{ \pm C}$

(f)

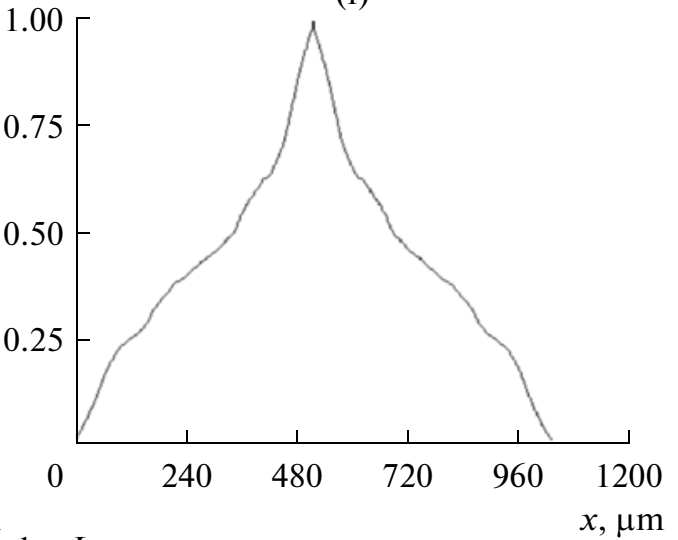

$\log J_{ \pm C}$

(h)

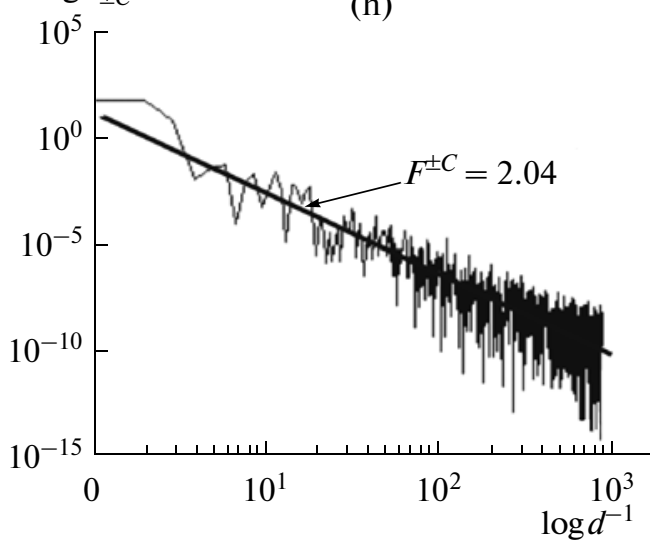

Fig. 4. Coordinate $V_{4}(m \times n)=1\left((\mathrm{a})\right.$, (b)), and quantitative $N_{L}(x)$ distributions ((c), (d)), of $\pm C$-states in polarization; autocorrelation functions $K_{ \pm C}(\Delta m)$ (e), (f), and $\log J_{L}-\log d^{-}$dependencies ((g), (h)) for power spectra $J\left(N_{ \pm C}\right)$ of the distribution $N_{ \pm C}(x)$ for laser images of the of the different types of PhILS 2-3. 
Table 1. Statistical, correlation and fractal parameters for the distribution of the amount of polarization-singular states in laser images of PhILS

\begin{tabular}{l|c|c|c|c|c|c}
\hline \multirow{2}{*}{$\begin{array}{l}\text { Parameters } \\
(L-; \pm C-)\end{array}$} & \multicolumn{2}{|c|}{ PhILS-1 } & \multicolumn{2}{c|}{ PhILS-2 } & \multicolumn{2}{c}{ PhILS-3 } \\
\cline { 2 - 7 } & $L-$ & $\pm C-$ & $L-$ & $\pm C-$ & $L-$ & $\pm C-$ \\
\hline$Z_{1}$ & $0.7 \pm 0.075$ & - & $0.42 \pm 0.045$ & $0.13 \pm 0.032$ & $0.23 \pm 0.034$ & $0.16 \pm 0.034$ \\
$Z_{2}$ & $0.12 \pm 0.023$ & - & $0.22 \pm 0.04$ & $0.35 \pm 0.049$ & $0.32 \pm 0.051$ & $0.28 \pm 0.039$ \\
$Z_{3}$ & $0.05 \pm 0.007$ & - & $0.07 \pm 0.009$ & $0.44 \pm 0.057$ & $0.03 \pm 0.006$ & $0.05 \pm 0.008$ \\
$Z_{4}$ & $0.18 \pm 0.034$ & - & $0.08 \pm 0.011$ & $0.56 \pm 0.083$ & $0.08 \pm 0.009$ & $0.11 \pm 0.016$ \\
$S$ & $0.28 \pm 0.02$ & - & $0.24 \pm 0.021$ & $0.18 \pm 0.052$ & $0.22 \pm 0.031$ & $0.24 \pm 0.027$ \\
$Q$ & $0.09 \pm 0.01$ & - & $0.11 \pm 0.089$ & $1.16 \pm 0.015$ & $0.17 \pm 0.022$ & $0.11 \pm 0.017$ \\
$F$ & $2.02 \pm 0.11$ & - & $2.12 \pm 0.016$ & - & $2.31 \pm 0.09$ & $2.07 \pm 0.13$ \\
$D$ & $0.22 \pm 0.053$ & - & $0.24 \pm 0.017$ & $0.41 \pm 0.052$ & $0.27 \pm 0.038$ & $0.23 \pm 0.036$ \\
\hline
\end{tabular}

This fact is indicative of another, more complex, statistical distruibution for the amount of $\pm C$ polarization states as compared with the equiprobable distribution of $L$ polarization states in laser images of the PhILS-2.

The autocorrelation function $K^{ \pm C}(\Delta m)$ of the dependence $N_{ \pm C}(x)$ (Fig. 4e) rapidly drops with increasing the scanning step $\Delta m$, which shows the random distribution of states with circular polarization in laser images of the ground PhILS-2. The correlation area $S^{ \pm C}$ and correlation moment $Q^{ \pm C}$ of the distribution $N_{ \pm C}(x)$ as compared with similar correlation parameters of $N_{L}(x)$ distributions (Fig. $3 \mathrm{~h}$ ) experience changes in inverse proportion: $S^{ \pm C}(\downarrow)=0.165$ and $Q^{ \pm C}(\uparrow)=0.95$.

The random character of the $N_{ \pm C}(x)$ distribution is also confirmed by the absence of a stable value for the slope angle of the approximating curve to the logarithmic dependence $\log J_{ \pm C}-\log d^{-1}$ (Figs. 4g, 4h). In this case, the dispersion value $D^{ \pm C}$ grows up to 2.15 times as compared with the data obtained for logarithmic dependences of power spectra for distributions of the amount of linearly polarized states: $D^{ \pm C}=0.49$.

The phase modulation of multiply scattered laser radiation by the PhILS-3 is characterized by the network of $\pm C$-states (Fig. 4b).

The total amount of $L$ - and $\pm C$-states of polarization for the respective laser image is approximately the same (Figs. 3f, $4 \mathrm{~d}$ ). Like to the case of $N_{L}(x)$ distribution for the amount of linearly polarized states, the $N_{ \pm C}(x)$ distribution is close to the equiprobable one: $Z_{j=3 ; 4}^{ \pm C} \ll Z_{j=1 ; 2}^{ \pm C}$. Differences between statistical moments $Z_{j=1 ; 2 ; 3 ; 4}^{L}$ and $Z_{j=1 ; 2 ; 3 ; 4}^{ \pm C}$ are insignificant and lie within 25 to $35 \%: Z_{1}^{ \pm C}=0.145 ; Z_{2}^{ \pm C}=0.33$; $Z_{3}^{ \pm C}=0.04 ; Z_{4}^{ \pm C}=0.09$.

The values of the correlation area $S^{ \pm C}$ and correlation moment $Q^{ \pm C}$ are close to their extremum ones: $S^{ \pm C}=0.25 ; Q^{ \pm C}=0.1$.

Our analysis of the logarithmic dependences (Fig. 4h) for the power spectra $J\left(N_{ \pm C}\right)$ of the $N_{ \pm C}(x)$ distribution in laser images of the PhILS-3 found a stable slope of the approximating curve: $F^{ \pm C}=2.04$. The dispersion value of the distribution $\log J_{ \pm C}-\log d^{-1}$ is 1.7 times grown as compared with the analogous statistical moment that characterizes the dependence $\log J_{L}-\log d^{-1}\left(D^{ \pm C}=0.24\right)$.

\section{POLARIZATION-SINGULAR CLASSIFICATION AND DIFFERENTIATION OF OPTICAL PROPERTIES INHERENT TO PHILS}

As the objects of investigation the fifteen histological sections of skin for all the groups (PhILS 1 to 3) were chosen:

-PhILS $1(\tau<0.1)$, which were obtained from the different points of skin surface;

-PhILS $2(\tau=0.2-0.4)$;

-PhILS $3(\tau=0.5 ; 0.85 ; 1.25 ; 1.8 ; 2.3)$.

The statistically averaged (within the limits of groups 1 to 3 ) values and ranges of changing statistical moments $Z_{j=1 ; 2 ; 3 ; 4}^{L ; C}$, correlation $Q^{L ; \pm C}, S^{L ; \pm C}$ and fractal $F^{L ; \pm C}, D^{L ; \pm C}$ parameters that characterize the $N_{ \pm C}(x)$ dependences for the amount of singular states in laser images of PhILS have been illustrated on Table 1 . 
Table 2. Polarization-singular differentiation of optical properties of PhILS

\begin{tabular}{c|c|c}
\hline PhILS & \multicolumn{2}{c}{ Groups 1-3 } \\
\hline Parameters & $N_{L}$ & $N_{ \pm C}$ \\
\hline$Z_{1}$ & $\oplus$ & $\oplus$ \\
$Z_{2}$ & $\oplus$ & $\oplus$ \\
$Z_{3}$ & $\otimes$ & $\oplus$ \\
$Z_{4}$ & $\otimes$ & $\oplus$ \\
$S$ & $\otimes$ & $\oplus$ \\
$Q$ & $\otimes$ & $\oplus$ \\
$F$ & $\otimes$ & $\oplus$ \\
\hline
\end{tabular}

Note: $\otimes$-differentiation is impossible; $\oplus$ — possible.

The performed analysis of results adduced in Table 1 for statistical $\left(Z_{j=1 ; 2 ; 3 ; 4}^{L ; \pm C}\right.$, correlation $\left(S^{L ; \pm C} ; Q^{L ; \pm C}\right)$ and fractal $\left(F^{L ; \pm C} ; D^{L ; \pm C}\right)$ parameters has shown:

\section{For PhILS of the group 1:}

- differentiation of optical properties inherent to rough surfaces with different micro-relief parameters epithelium layer is possible when using determination of values for the 2-nd and 4-th statistical moments of the distribution specific to the amount of $L$ polarization states: with increasing the micro-relief, the value $Z_{2}^{L}$ grows by 1.9 times, while the value $Z_{4}^{L}$ is 2.23 times lowered;

- the dispersion value $D^{L}$ for the distribution of extremes in logarithmic dependences $\log J_{L}-\log d^{-1}$ describing laser images of large-scale epithelium rough surfaces is 2.12 times increased;

- the values of correlation parameters $\left(S^{L ; \pm C} ; Q^{L ; \pm C}\right)$ that characterize the dependences $N_{L}(x)$ for laser images of surfaces with various sizes of micro-roughness (from 1 to $40 \mu \mathrm{m}$ ) cannot serve as reliable criteria for their differentiation.

\section{For PhILS of the group 2:}

- the variations of values inherent to all the statistical moments $Z_{j=1 ; 2 ; 3 ; 4}^{ \pm C}$ that characterize the distribution of the amount of $\pm C$-states in the respective laser images were found to be sensitive to changes in the thickness of top and subsurface layers on surfaces grow, $Z_{1}^{ \pm C}$ is 1.6 times decreased; $Z_{2}^{ \pm C}$ is 2.1 times increased; $Z_{3}^{ \pm C}$ increases by 1.78 times and $Z_{4}^{ \pm C}$ is 3.12 times increased;

- there observed are significant differences between the values of correlation areas $S^{ \pm C}(1.7$ times growth) and correlation moments $Q^{ \pm C}$ (2.89 times drop);

$-N^{ \pm C}$ distributions for the amount of $\pm C$-states in laser images of all the layers are statistical-the approximating curves for the dependences $\log J_{ \pm C}-\log d^{-1}$ have no stable slope;

- the dispersion value $D^{ \pm C}$ for the logarithmic dependences of power spectra for the $N^{ \pm C}$ distributions is changed insignificantly (by 1.12 times) and cannot serve as a reliable criterion for differentiation of optical properties of ground surfaces.

\section{For PhILS of the group 3:}

- there exists a possibility to use statistical moments of the 4-th order that characterize the $N^{L}(x)$ and $N^{ \pm C}(x)$ distributions for differentiation of optically-thick layers with a bulk scattering: with increasing the optical thickness, the differences between $Z_{4}^{L}$ and $Z_{4}^{ \pm C}$ reach 6 and 5 times, respectively;

-weak differences take place between the values of correlation parameters $S^{L ; \pm C} ; Q^{L ; \pm C}: 1.23$ and 1.36 times, respectively;

$-N^{L}(x)$ and $N^{ \pm C}(x)$ distributions are fractal.

\section{For PhILS of all the groups:}

The possibility to differentiate "group" optical properties of PhILS with surface, subsurface and bulk light scattering is illustrated in Table 2. 


\section{CONCLUSIONS}

(1) Analyzed in this work are the main physical mechanisms providing formation of polarization singularities in laser images of PhILS with surface, subsurface and bulk light scattering.

(2) Offered are statistical, correlation and fractal parameters for polarization-singular estimating the optical properties inherent to PhILS of all types.

(3) Determined are the ranges for changing the set of criteria that characterize distributions of the amount of polarization-singular states in laser images, which enabled us to realize both "intergroup" classification and "intragroup" differentiation of optical properties related to PhILS of various types.

\section{REFERENCES}

1. Xueding Wang, Gang Yao, and Lihong V. Wang, Monte Carlo Model and Single-Scattering Approximation of Polarized Light Propagation in Turbid Media Containing Glucose, Appl. Opt., 2002, vol. 41, pp. 792-801.

2. Xueding Wang and Lihong V. Wang, Propagation of Polarized Light in Birefringent Turbid Media: a Monte Carlo Study, J. Biomed. Opt., 2002, vol. 7, pp. 279-290.

3. Gang Yao and Lihong V. Wang, Two-Dimensional Depth-Resolved Mueller Matrix Characterization of Biological Tissue by Optical Coherence Tomography, Opt. Lett., 1999, vol. 24, pp. 537-539.

4. Demos, S.G. and Alfano, R.R., Optical Polarization Imaging, Appl. Opt., 1997, vol. 36, pp. 150-155.

5. Alexander G. Ushenko and Vasilii P. Pishak, Laser Polarimetry of Biological Tissue: Principles and Applications, in Handbook of Coherent-Domain Optical Methods: Biomedical Diagnostics, Environmental and Material Science, Valery V. Tuchin, Ed., Kluwer Academic, 2004, vol. 1, pp. 93-138.

6. Johannes F. de Boer, Thomas E. Milner, Martin J. C. van Gemert, and J. Stuart Nelson, Two-Dimensional Birefringence Imaging in Biological Tissue by Polarization-Sensitive Optical Coherence Tomography, Opt. Lett., 1997, vol. 22, pp. 934-936.

7. Shuliang Jiao, Gang Yao, and Lihong V. Wang, Depth-Resolved Two-Dimensional Stokes Vectors of Backscattered Light and Mueller Matrices of Biological Tissue Measured with Optical Coherence Tomography, Appl. Opt., 2000, vol. 39, pp. 6318-6324.

8. Shuliang Jiao and Lihong V. Wang, Two-Dimensional Depth-Resolved Mueller Matrix of Biological Tissue Measured with Double-Beam Polarization-Sensitive Optical Coherence Tomography, Opt. Lett., 2002, vol. 27, pp. 101-103.

9. Wang, X., Wang, L.-H., Sun, C.-W., and Yang, C.C., Polarized Light Propagation Through the Scattering Media: Time-Resolved Monte Carlo and Experiments, J. Biomed. Opt., 2003, vol. 8, pp. 608-617.

10. de Boer, J.F. and Milner, T.E., Review of Polarization Sensitive Optical Coherence Tomography and Stokes Vector Determination, J. Biomed. Opt., 2002, vol. 7, pp. 359-371.

11. Ducros, M.G., de Boer, J.F., Huang, H.E., Chao, L.C., Chen, Z.P., Nelson, J.S., Milner, T.E., and Rylander, H.G., Polarization Sensitive Optical Coherence Tomography of the Rabbit Eye, IEEE J. Select. Top. Quant. Electron., 1999, vol. 5, pp. 1159-1167.

12. de Boer, J.F., Milner, T.E., and Nelson, J.S., Two Dimensional Birefringence Imaging in Biological Tissue Using Phase and Polarization Sensitive Optical Coherence Tomography in Trends in Optics and Photonics (TOPS): Advances in Optical Imaging and Photon Migration, Washington: OSA, 1998.

13. Everett, M.J., Shoenenberger, K., Colston, B.W., and da Silva, L.B., Birefringence Characterization of Biological Tissue by Use of Optical Coherence Tomography, Opt. Lett., 1998, vol. 23, pp. 228-230.

14. Ushenko, A.G., Misevich, I.Z., Istratiy, V., Bachyns'ka, I., Peresunko, A.P., Numan Omar Kamal, and Moiysuk, T.G., Evolution of Statistic Moments of 2D-Distributions of Biological Liquid Crystal Net Mueller Matrix Elements in the Process of Their Birefringent Structure Changes, Advances in Optical Technologies, 2010, Article ID 423145, 2010.

15. Angelsky, O.V., Ushenko, A.G., and Ushenko, Ye.G., Investigation of the Correlation Structure of Biological Tissue Polarization Images During the Diagnostics of Their Oncological Changes, Phys. Med. Biol., 2005, vol. 50, pp. 4811-4822.

16. Ushenko, A.G., Laser Polarimetry of Polarization-Phase Statistical Moments of the Object Field of Optically Anisotropic Scattering Layers, Optics Spectrosc., 2001, vol. 91, pp. 313-316.

17. Ushenko, A.G., Correlation Processing and Wavelet Analysis of Polarization Images of Biological Tissues, Optics and Spectroscopy, 2002, vol. 91, pp. 773-778.

18. Dubolazov, O.V., Ushenko, A.G., Bachynsky, V.T., Peresunko, A.P., and Vanchulyak, O.Ya., On the Feasibilities of Using the Wavelet Analysis of Mueller Matrix Images of Biological Crystals, Advances in Optical Technologies, 2010, Article ID 162832, 2010.

19. Angelsky, O.V., Maksimyak, P.P., and Perun, T.O., Dimensionality in Optical Fields and Signals, Appl. Opt., 1993, vol. 32, pp. 6066-6071. 
20. Angelsky, O.V., Maksimyak, P.P., and Hanson, S., The Use of Optical-Correlation Techniques for Characterizing Scattering Object and Media, Bellingham: SPIE Press PM71, 1999.

21. Angelsky, O.V., Maksimyak, P.P., and Perun, T.O., Optical Correlation Method for Measuring Spatial Complexity in Optical Fields, Opt. Lett., 1993, vol. 18, pp. 90-92.

22. Angelsky Oleg. V., Burkovets Dmitriy N., Kovalchuk Alexander V., and Hanson Steen G., Fractal Description of Rough Surfaces, Appl. Opt., 2002, vol. 41, pp. 4620-4629.

23. Freund Isaac, Soskin Marat S., and Mokhun Alex I., Elliptic Critical Points in Paraxial Optical Fields, Optics Commun., 2002, vol. 207, pp. 223-253.

24. Soskin, M.S., Denisenko, V., and Freund, I., Optical Polarization Singularities and Elliptic Stationary Points, Opt. Lett., 2003, vol. 28, pp. 1475-1477.

25. Dennis, M.R., Polarization Singularities in Paraxial Vector Fields: Morphology and Statistics, Opt. Commun., 2002, vol. 213, pp. 201-221.

26. Soskin, M.S., Denisenko, V.G., and Egorov, R.I., Singular Elliptic Light Fields: Genesis of Topology and Morphology, Proc. SPIE., 2006, vol. 6254, p. 625404.

27. Nye, J.F., Lines of Circular Polarization in Electromagnetic Wave Fields, Proc. R. Soc. London, Ser. A., 1983, vol. 389, pp. 279-290.

28. Flossmann, F., Schwarz, U.T., Maier, M., and Dennis, M.R., Polarization Singularities from Unfolding an Optical Vortex Through a Birefringent Crystal, Phys. Rev. Lett., 2005, vol. 95, p. 253901.

29. Flossmann, F., Schwarz, U.T., Maier, M., and Dennis, M.R., Stokes Parameters in the Unfolding of an Optical Vortex Through a Birefringent Crystal, Opt. Express., 2006, vol. 14, pp. 11402-11411.

30. Bliokh, K.Y., Niv, A., Kleiner, V., and Hasman, E., Singular Polarimetry: Evolution of Polarization Singularities in Electromagnetic Waves Propagating in a Weakly Anisotropic Medium, Opt. Express., 2008, vol. 16, pp. 695709.

31. Angelsky, O., Besaha, R., Mokhun, A., Mokhun, I., Sopin, M., Soskin, M., and Vasnetsov, M., Singularities in Vectoral Fields, SPIE Proc., 1999, vol. 3904, pp. 40-55.

32. Freund, I., Mokhun, A.I., Soskin, M.S., Angelsky, O.V., and Mokhun, I.I., Stokes Singularity Relations, Opt. Let., 2002, vol. 27, pp. 545-547.

33. Angelsky, O., Mokhun, A., Mokhun, I., and Soskin, M., The Relationship between Topological Characteristics of Component Vortices and Polarization Singularities, Optics Commun., 2002, vol. 207, pp. 56-57.

34. Berry, M.V. and Dennis, M.R., Polarization Singularities in Isotropic Random Vector Waves, Proc. R. Soc. Lond. A., 2001, vol. 457, pp. 141-155.

35. Angelsky Oleg V., Ushenko Alexander G., Ushenko Yevheniya G., and Tomka Yuriy Y., Polarization Singularities of Biological Tissues Images, J. Biomed. Opt., 2006, vol. 11, p. 054030.

36. Angelsky, O.V., Ushenko, A.G., Ushenko, Yu.A., and Ushenko, Ye.G., Polarization Singularities of the Object Field of Skin Surface, J. Phys. D: Appl. Phys., 2006, vol. 39, pp. 3547-3558.

37. Angelsky, O.V., Ushenko, A.G., Angelska, A.O., and Ushenko, Yu.A., Correlation- and Singular-Optical Approaches in Diagnostics of Polarization Inhomogeneity of Coherent Optical Fields from Biological Tissues, Ukr. J. Phys. Opt., 2007, vol. 8, pp. 105-123.

38. Ushenko, A.G., Stokes-Correlometry of Biotissues, Laser Phys., 2000, vol. 10, pp. 1286-1292. 